\title{
Effect of Sensory Stimuli on Dynamic Loading Induced by People Bouncing
}

\author{
Vitomir Racic ${ }^{1}$, James M. W. Brownjohn ${ }^{2}$, Shu Wang ${ }^{3}$, Mark T. Elliot ${ }^{4}$, Alan Wing ${ }^{5}$ \\ ${ }^{1}$ Lecturer, ${ }^{2}$ Professor, ${ }^{3}$ Research Assistant - Vibration Engineering Section \\ Department of Civil and Structural Engineering, The University of Sheffield \\ Sir Frederick Mappin Building, Mappin Street, Sheffield S1 3JD, United Kingdom \\ ${ }^{4}$ Research Assistant, ${ }^{5}$ Professor - Sensory Motor Neuroscience \\ Department of Psychology, The University of Birmingham \\ Edgbaston, Birmingham, B15 2TT, United Kingdom
}

\begin{abstract}
Prediction of dynamic loads induced by groups and crowds of people bouncing is a hot topic among designers of grandstands and floors in entertaining venues. Using motion capture technology transferred and adapted from biomedical research, this study aims to investigate effect of visual, auditory and tactile cues on the ability of people to coordinate or synchronise their bouncing movements in groups of two. The numerical results showed a great significance of such stimuli on people's mutual interaction during bouncing, signifying that their effect should be considered in developing much-needed models of crowd dynamic loading of structures due to coordinated rhythmic activities.
\end{abstract}

KEYWORDS: vibration serviceability, ground reaction forces, synchronisation, entertaining venues, grandstands.

\section{NOMENCLATURE}

$\begin{array}{clcl}\alpha & \text { coefficient of synchronisation } & T_{b} & \text { average duration of bouncing cycle } \\ \Delta_{\mathrm{i}} & \text { time lag } & \mathrm{n} & \text { number of bouncing cycles }\end{array}$

\section{INTRODUCTION}

In the last decade, a number of newly built entertainment venues have experienced vibration serviceability issues when occupied and dynamically excited by groups of active people during rock concerts [1-2]. There are three key reasons for this happening:

(1) lightness of modern structural materials yields high human to structural mass ratio [3],

(2) natural frequency of the structure and frequency of human-induced loading are close [3], and

(3) dancing to a steady rhythm of the rock music coupled with close proximity between individuals, helps the occupants to synchronise vertical motion of their bodies leading to large excitation amplitudes [4-5].

Since the proximity between individuals in groups also limits their horizontal movements, dancing featuring rock concerts can be reliably described as bouncing in place, i.e. a cyclic activity similar to jumping but with the both feet in permanent contact with the ground [3]. This study aims to investigate the effect of auditory, visual and tactile stimuli (also called cues) on the ability of people to synchronise their bouncing movements in groups of two. Section 2 describes experimental data collection. Section 3 presents a simple numerical measure of the synchronisation effect for different combinations of the stimuli, while Section 4 is a summary of the key results and findings of this study. 


\section{DATA COLLECTION}

Two Codamotion [6] tracking cameras (Figure 1) were used to record vertical positions of two motion tracking markers attached to the neck of each test subject (Figure 2). The markers were arranged in the way to prevent occlusions between test subjects, i.e. at least one tracking marker per test subject was visible during the experiment.

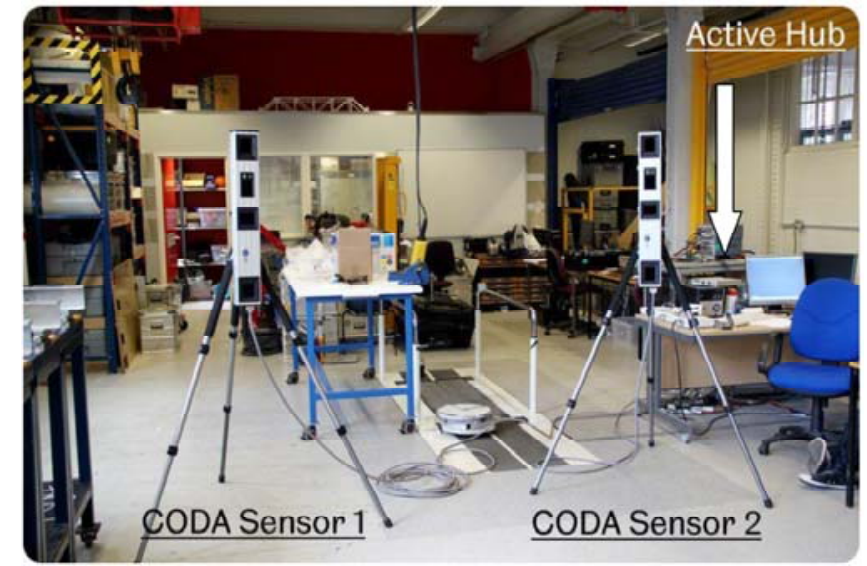

Figure 1: Experimental setup at Vibration Engineering Section Laboratory at the University of Sheffield.

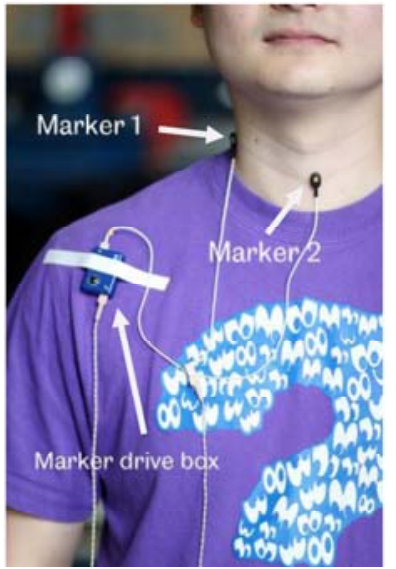

Figure 2: Tracking markers attached to the neck.

Eight healthy male volunteers participated in the experiments. They were split into four pairs and each pair was independently asked to follow a steady beep provided by an electronic metronome. Metronome rates $1.3 \mathrm{~Hz}, 2.0 \mathrm{~Hz}$ and $2.7 \mathrm{~Hz}$ were selected to represent audio stimuli corresponding to slow, moderate and fast rock music rhythms [7].

To test the hypothesis that possibility to see each other improves the synchronisation of the pairs, test protocol included three different bouncing positions:

1) side-by-side: participants could use only their peripheral vision,

2) facing: participants could make full eye contact when bouncing, and

3) back-to-back: participants could not see each other.

The tests designed to investigate the effect of proximity between two bouncing bodies on their synchronisation included close, medium $(0.5 \mathrm{~m})$ and far $(1 \mathrm{~m})$ distances between individuals, as shown in Figure 3 . Finally, the influence of tactile cues was investigated in tests where participants were bouncing close together with and without holding their hands.

Each pair completed 33 tests based on a number of combinations between different bouncing rates, proximity between individuals and visual and tactile cues. Each test was repeated three times to ensure statistical reliability. An example of recorded the position time histories is given in Figure 4.
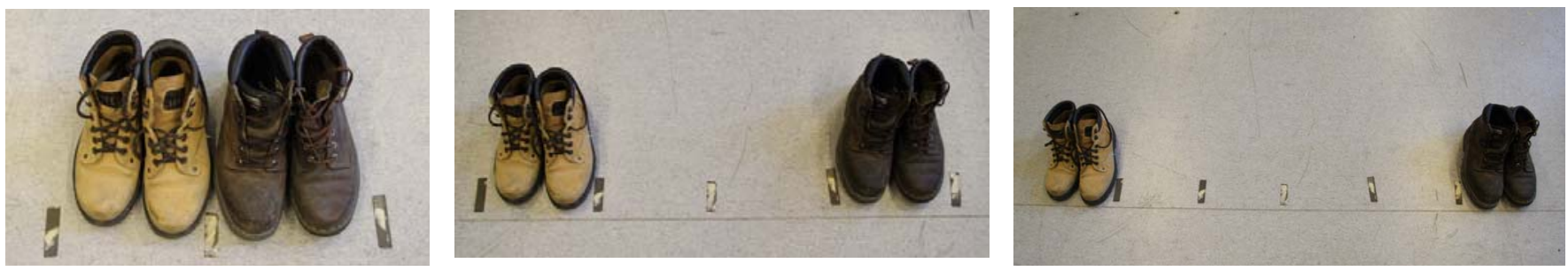

Figure 3: Close, medium and far distance between individuals. 


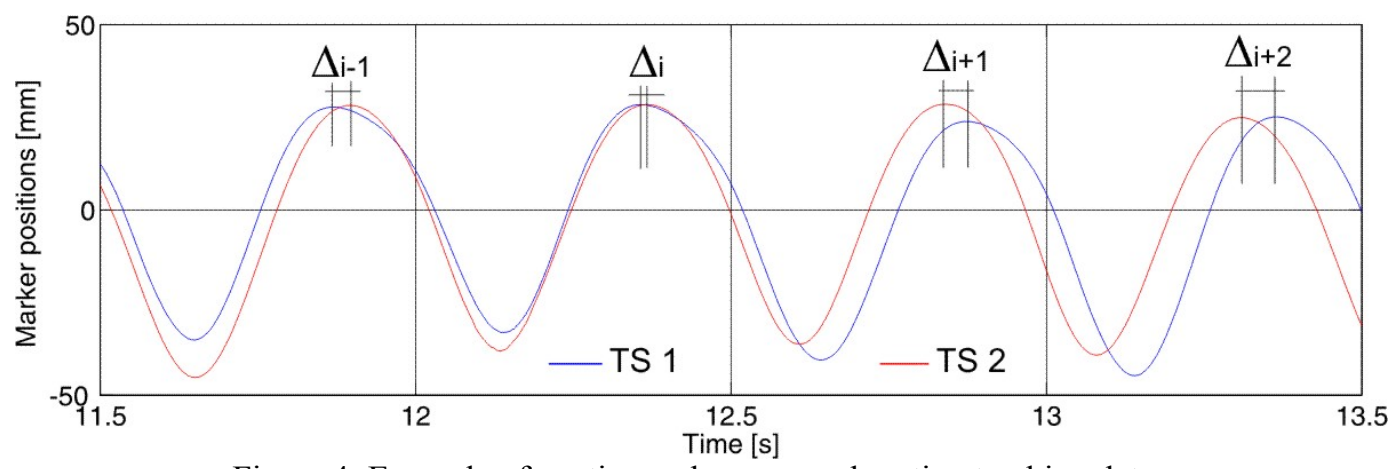

Figure 4: Example of continuously measured motion tracking data.

\section{DATA ANALYSIS}

Using the motion data of a kind presented in Figure 4, coefficient of synchronisation $\alpha$ can be calculated as:

$$
\alpha=\frac{\sum_{\mathrm{i}=1}^{\mathrm{n}}\left|\Delta_{\mathrm{i}}\right|}{\mathrm{nT}_{\mathrm{b}}}=\frac{\mathrm{f}_{\mathrm{b}}}{\mathrm{n}} \sum_{\mathrm{i}=1}^{\mathrm{n}}\left|\Delta_{\mathrm{i}}\right|
$$

Here, $\Delta_{\mathrm{i}}$ is a time lag between the body positions during $\mathrm{i}$-th bouncing cycle, $\mathrm{n}$ is the total number of successive bouncing cycles in the motion signals, $T_{b}$ is an average period of the bouncing cycle and $f_{b}$ is the bouncing frequency. The coefficient was calculated for all tests and the results were averaged between the pairs for tests with the same combination of metronome beats and visual, spatial and tactile cues. The results are illustrated in Figures 5-7 and the key findings are listed in the next section.

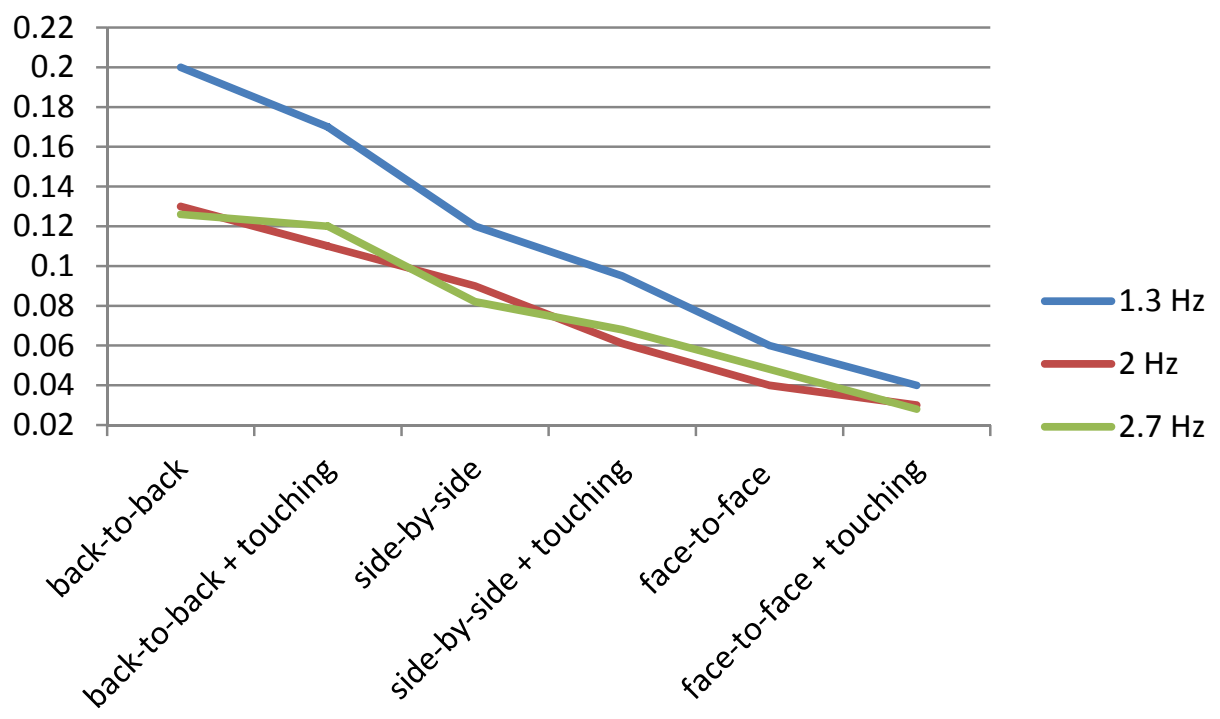

Figure 5: Average $\alpha$ values for tests featuring close distance between individuals bouncing. 


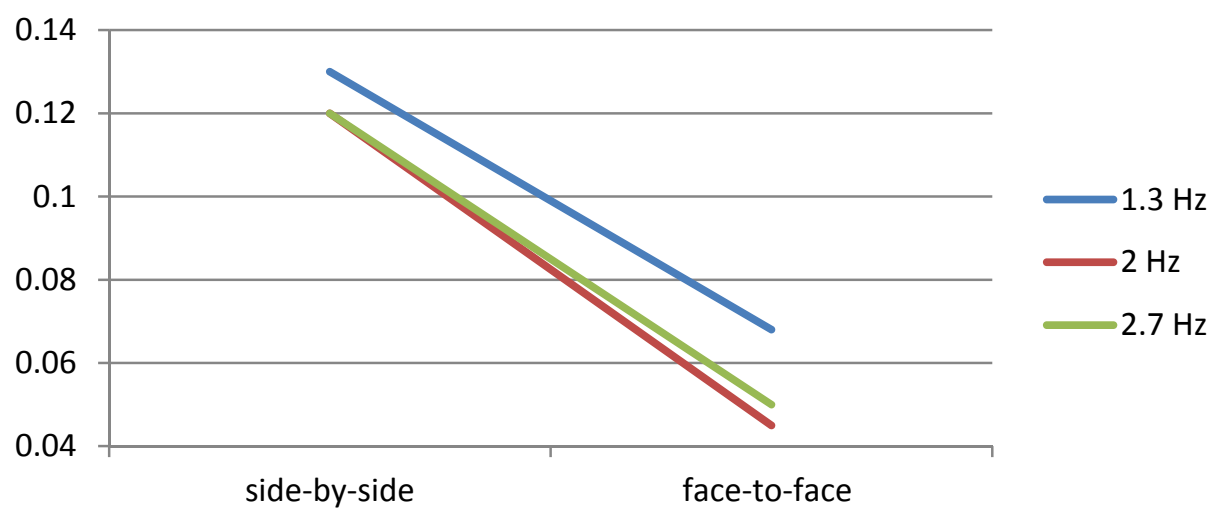

Figure 6: Average $\alpha$ values for tests featuring medium distance between individuals bouncing.

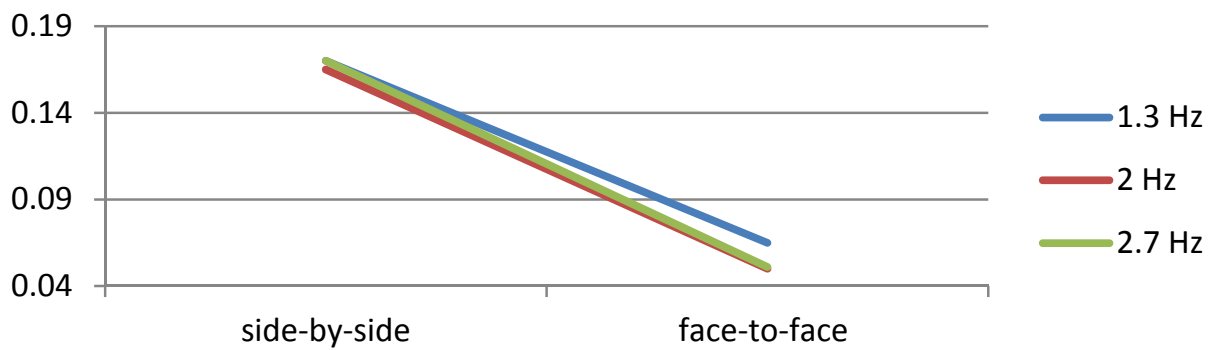

Figure 7: Average $\alpha$ values for tests featuring far distance between individuals bouncing.

\section{RESULTS AND CONCLUSIONS}

The key findings of this study are:

- Audio, visual and tactile stimuli can considerably improve synchronisation between two persons bouncing. Therefore, a reliable prediction of the structural response due to groups bouncing should include a reliable model of the synchronisation effect.

- For the given audio stimuli, the synchronisation improves with decreasing proximity between people and possibility to see and touch each other. Hence, pairs are best synchronised when facing each other and holding hands. On the other hand, metronome stimuli alone (i.e. in back-to-back tests and no touching) resulted in the lowest level of synchronisation.

- For the same combination of audio, visual and tactile cues, in the majority of cases the best synchronisation was achieved when bouncing at $2 \mathrm{~Hz}$. This is probably because the energy consumption at this rate was lower than when bouncing at $1.3 \mathrm{~Hz}$ and $2.7 \mathrm{~Hz}$. In feedback after the experimental data collection, all participants reported that bouncing at $1.3 \mathrm{~Hz}$ was the most tiring and that $2.7 \mathrm{~Hz}$ rate felt slightly less comfortable than $2 \mathrm{~Hz}$. This is clearly reflected in the results shown in Figures 5-7.

Recommendations for future research:

- Tests should be carried out in natural environments, such as real concert events, rather than in constrained laboratory settings.

- Metronome beats should be replaced with music to simulate reality better.

- Extended experimental data collection should include tests with groups of various sizes. The so established database will make possible to learn if synchronisation changes with increasing number of people, leading to reliable predictions of the synchronisation effect for large groups and ultimately crowds.

- A wider range of bouncing frequencies should be tested, including frequencies up to $4 \mathrm{~Hz}$ which are becoming increasingly present in the popular contemporary music.

\section{REFERENCES}

[1] Glackin M (2000) Stadia design rethink prompted by Cardiff fiasco. Building, pg. 11.

[2] Parker D (2003) Rock fans uncover town hall floor faults. New Civil Engineer, 20 November. 
[3] Jones CA, Reynolds P and Pavic A (2011) Vibration serviceability of stadia structures subjected to crowd loads: a literature review. Journal of Sound and Vibration 330 (8), 1531-1566.

[4] Ebrahimpour A and Fitts LL (1996) Measuring coherency of human-induced rhythmic loads using force plates. ASCE Journal of Structural Engineering 122 (7), 829-831.

[5] Luck G and Sloboda JA (2009) Spatio-temporal cues for visually mediated synchronization. Music Perception 26 (5), 465-473.

[6] Codamotion user manuals, Charnwood Dynamics Ltd., Leicestershire, UK, 2012.

[7] Littler JD (2003) Frequencies of synchronised human loading from jumping and stamping. The Structural Engineer 22, 27-35. 